\title{
Serum carboxy-terminal telopeptide of type I collagen levels are associated with carotid atherosclerosis in patients with cardiovascular risk factors
}

\author{
Takeshi Kondo ${ }^{1)}$, Itsuro Endo ${ }^{1)}$, Ken-ichi Aihara ${ }^{1)}$, Yukiyo Onishi ${ }^{1)}$, Bingzi Dong ${ }^{1)}$, Yukari Ohguro'), \\ Kiyoe Kurahashi $^{1)}$, Sumiko Yoshida ${ }^{1)}$, Yuichi Fujinaka ${ }^{2}$, Akio Kuroda ${ }^{3)}$, Munehide Matsuhisa ${ }^{3)}$, \\ Seiji Fukumoto ${ }^{4)}$, Toshio Matsumoto ${ }^{4)}$ and Masahiro Abe ${ }^{1)}$ \\ 1) Department of Hematology, Endocrinology \& Metabolism Institute of Biomedical Sciences, Tokushima University Graduate \\ School, Tokushima 770-8503, Japan \\ 2) Department of Internal Medicine, Local Incorporated Administrative Agency, Tokushima Prefecture Naruto Hospital, Naruto \\ 772-0001, Japan \\ 3) Diabetes Therapeutics and Research Center, Tokushima University, Tokushima 770-8503, Japan \\ 4) Fujii Memorial Institute of Medical Sciences, Tokushima University, Tokushima 770-8503, Japan
}

\begin{abstract}
Carboxy-terminal telopeptide of type I collagen (ICTP) is generated through matrix metalloproteinase (MMP)dependent type I collagen digestion, and has been widely utilized as a biomarker for bone turnover. The fact that atherosclerotic lesions are rich in both type I collagen and MMP-producing macrophages led to the hypothesis that serum ICTP concentrations may serve as a non-invasive clinical biomarker for atherosclerosis. Therefore, the association of serum ICTP concentrations with the maximum intima-media thickness (IMT) of carotid arteries, a surrogate index of systemic atherosclerosis, or brachial-ankle pulse wave velocity (baPWV) in patients with atherosclerotic risk factors was evaluated. A total of 52 male and 65 female (mean age: $62.8 \mathrm{yrs}$ ) patients without renal failure, malignancies or bone diseases known to affect serum ICTP concentrations were recruited. Patients with max IMTs $\geq 1.1 \mathrm{~mm}$ showed significantly higher serum ICTP concentrations compared with patients with max IMTs $<1.1 \mathrm{~mm}(3.33 \pm 0.97$ vs $2.82 \pm 0.65 \mathrm{ng} / \mathrm{mL}$, $p<0.05)$. Serum ICTP concentration was also positively correlated with max IMT $(p<0.001)$ or baPWV values $(p<0.05)$. Multivariate analyses also revealed that serum ICTP concentrations were correlated with max IMT $(p<0.001 ; 95 \%$ CI 0.200 to 0.454 ). These results suggest that serum ICTP concentrations can be used as a non-invasive biomarker for systemic atherosclerosis.
\end{abstract}

Key words: ICTP, Atherosclerosis, Max IMT, baPWV

SYSTEMIC atherosclerosis is closely associated with incidence of acute coronary syndrome, cerebrovascular ischemia and peripheral arterial diseases. The vascular health should be assessed in patients with cardiovascular risk factors to prevent progression of total vascular events. Measurement of maximum carotid intimamedia thickness (IMT) is widely used as a non-invasive and reliable surrogate marker for systemic atherosclerosis [1-3], and patients with max IMTs $\geq 1.1 \mathrm{~mm}$ has been demonstrated to have an increased risk of cardio-

Submitted Oct. 15, 2015; Accepted Jan. 6, 2016 as EJ15-0589 Released online in J-STAGE as advance publication Feb. 13, 2016 Correspondence to: Itsuro Endo, M.D., Ph.D., Department of Hematology, Endocrinology \& Metabolism Institute of Biomedical Sciences, Tokushima University Graduate School, Tokushima 7708503, Japan. E-mail: endoits@tokushima-u.ac.jp vascular events compared to those with max IMTs $<1.1$ $\mathrm{mm}$ [2]. Brachial-ankle pulse wave velocity (baPWV) is also utilized as a non-invasive index of systemic atherosclerosis $[4,5]$.

Type I collagen is known as a major component of collagens localized in the intima, media and adventitia of blood vessel walls [6]. Abnormal metabolism of a matrix collagen turnover in a vessel wall eventually causes arterial stiffness [7]. In addition, circulating levels of type I collagen-derived peptides such as amino-terminal propeptide of procollagen type I (PINP) have been proposed as a useful biomarker to estimate the levels of cardiovascular fibrosis leading to cardiovascular events [8]. Besides PINP, carboxy-terminal telopeptide of type I collagen (ICTP) has also been attracting considerable attention, and we previ- 
ously demonstrated that serum ICTP levels could be a non-invasive biomarker for the formation of vulnerable plaques in coronary arteries [9]. Because atherosclerotic lesions with vulnerable plaques are rich in type I collagen and macrophages producing matrix metalloproteinases (MMPs) [10,11], type I collagen-degraded products by MMPs may be abundantly formed in the lesions. Because atherosclerosis progresses concomitantly in a wide variety of arteries [12, 13], ICTP formation may be systematically up-regulated as atherosclerosis progresses. Therefore, the present study was undertaken to determine whether serum ICTP levels can be used as a surrogate biomarker for the development of systemic atherosclerosis.

\section{Patients and Methods}

We recruited patients who visited to Tokushima University Hospital due to lifestyle-related diseases between March 2008 and April 2013. After taking a written informed consent, we evaluated atherosclerotic risk factors, including hypertension, dyslipidemia, diabetes mellitus, and current smoking. Patients with at least one of the atherosclerotic risk factors described above were enrolled in this study. We excluded patients with overt renal dysfunction, metabolic bone diseases, thyroid diseases or known malignancies which may affect serum ICTP levels. Blood samples were taken before noon from patients after overnight fasting, and serum levels of bone turnover markers including ICTP were measured. Data of work-up study for atherosclerosis were collected, including $\mathrm{HbA1c}$, triglyceride (TG), low density lipoprotein cholesterol (LDLC), high density lipoprotein cholesterol (HDL-C), lipoprotein(a) (Lp(a)) and high sensitivity C-reactive protein (hs-CRP). The levels of TG, LDL-C, HDL$\mathrm{C}, \mathrm{Lp}(\mathrm{a})$, hs-CRP and creatinine $(\mathrm{Cr})$ were measured by enzymatic methods. The estimated glomerular filtration rate (eGFR) was calculated using the following latest Japanese equation: eGFR $\left(\mathrm{mL} / \mathrm{min} / 1.73 \mathrm{~m}^{2}\right)=$ $194 \times \mathrm{Age}^{-0.287} \times \mathrm{Cr}^{-1.094}$ (if female, $\left.\times 0.739\right)$. HbAlc levels were assayed using latex agglutination. Serum ICTP levels were measured by a two-antibody telopeptide ICTP radioimmunoassay kit (Orion Diagnostica, Espoo, Finland). As bone turnover markers, serum levels of type I collagen cross-linked N-telopeptide (NTX) and bone-specific alkaline phosphatase (BSAP) were measured by enzyme-linked immunosorbent assays (Ostex International, Seattle, USA) and a chemilu- minescent enzyme immunoassay (Beckman Coulter, Bera, USA), respectively.

The baPWV was measured after resting for $15 \mathrm{~min}$ utes in the supine position in an air-conditioned room using a vascular testing device (Form PWV/ABI, Omron Healthcare, Kyoto, Japan). The four cuffs of a volume-plethysmographic device with oscillometric sensors were wrapped around the upper arms and ankles and then inflated automatically and simultaneously. The mean of the right and left baPWV values was used for the analysis. The validity and reproducibility of the measurements by this device had been assessed in previous studies [14, 15]. Ultrasound examinations were performed after 15 minutes of rest in the supine position using an ultrasound instrument with a 12-MHz B-mode transducer (Hitachi EUB8500, Hitachi Medical Corporation, Tokyo, Japan). The subject's head and sonographer's position and the scanning angles sequence were standardized. Bilateral common carotid arteries, carotid bifurcation and internal carotid arteries were scanned. The greatest intimamedia thickness (IMT) value was recorded as a max IMT $[16,17]$. All scans were performed by the same sonographer, and all image analyses were conducted by the same investigators who were blinded to the group assignment of the subjects.

For flow-mediated vasodilation (FMD) measurement, patients were rested for at least 15 minutes in the supine position. Using a 10-MHz linear array transducer probe positioned with a hybrid probe holder, longitudinal images of the right brachial artery were recorded at baseline, then a forearm-cuff was inflated for five minutes at $50 \mathrm{mmHg}$ above the systolic blood pressure just prior to FMD measurement [16]. Following cuff deflation, diastolic diameters of brachial arteries were monitored for 2 minutes using an instrument equipped with a software program (Unex Co. Ltd., Nagoya, Japan) [17]. FMD was then estimated as the percent change in the vessel diameter over the baseline value at maximal dilation during reactive hyperemia. The intraobserver variation of $\%$ FMD was $4.5 \%$.

The values are expressed as means $\pm \mathrm{SD}$ or as a percentage for categorical parameters. Serum ICTP levels between groups with and without a carotid plaque were compared by an unpaired $t$-test. Male gender and a presence of hypertension, diabetes mellitus, dyslipidemia, or current smoking were coded as dummy variables. All the variables were assessed by means of simple linear regression and stepwise or forced multiple 
regression analyses. These analyses were performed with SPSS statistics 17.0 for Macintosh (IBM SPSS Japan Inc., Tokyo, Japan). A $p$ value of less than 0.05 was considered to be statistically significant.

This study was approved by institutional review board of Tokushima University Hospital (No. 2012).

\section{Results}

\section{Patients' characteristics}

We enrolled 52 men and 65 women (41-82 years,

Table 1 Patient characteristics

\begin{tabular}{|c|c|c|c|}
\hline & & & Range \\
\hline Patien & mber & 117 & \\
\hline Gende & le/Female & $52 / 65$ & \\
\hline post 1 & pausal subject, $n /$ female & $53 / 65$ & \\
\hline Age (y & & $62.8 \pm 11.1$ & $41-82$ \\
\hline BMI (1 & & $24.6 \pm 4.03$ & $19.8-32.2$ \\
\hline Curren & king, n (\%) & $36(30.8)$ & \\
\hline Hyper & $n, n(\%)$ & $71(60.7)$ & \\
\hline Diabet & ellitus, n (\%) & $18(15.4)$ & \\
\hline Dyslip & ia, n (\%) & $71(60.7)$ & \\
\hline SBP $(1$ & & $133.0 \pm 19.6$ & $97-185$ \\
\hline DBP ( & & $77.5 \pm 12.8$ & $48-116$ \\
\hline $\mathrm{PP}(\mathrm{m}$ & & $55.4 \pm 14.8$ & $55-128$ \\
\hline TG $(\mathrm{m}$ & & $120.4 \pm 62.8$ & $30-352$ \\
\hline LDL-C & $/ \mathrm{dL})$ & $125.2 \pm 30.5$ & $47-194$ \\
\hline HDL-( & $3 / d L)$ & $60.8 \pm 16.4$ & $27-121$ \\
\hline $\mathrm{Lp}(\mathrm{a})$ & & $14.7 \pm 10.1$ & $0.2-58.1$ \\
\hline $\mathrm{Cr}(\mathrm{mg}$ & & $0.71 \pm 0.16$ & $0.42-1.00$ \\
\hline eGFR & Total & $74.9 \pm 17.8$ & $57.0-144.9$ \\
\hline & Male & $74.8 \pm 15.1$ & $66.3-113.6$ \\
\hline & Female & $74.5 \pm 18.8$ & $57.0-144.9$ \\
\hline $\mathrm{HbAl}$ & & $5.66 \pm 0.83$ & 4.4-9.1 \\
\hline hsCRF & (dL) & $0.20 \pm 1.15$ & $0.002-1.14$ \\
\hline baPW & a/sec) & $1,513.1 \pm 296.8$ & $987-2,748$ \\
\hline Max-I & $\mathrm{mm})$ & $1.60 \pm 0.83$ & $0.64-4.80$ \\
\hline$\%$ FMI & & $4.36 \pm 2.11$ & $0.2-10.3$ \\
\hline ICTP & & $3.21 \pm 0.93$ & $1.6-7.5$ \\
\hline
\end{tabular}

The values are presented as the mean \pm SD. Abbreviation: BMI, body mass index; SBP, systolic blood pressure; DBP, diastolic blood pressure; PP, pulse pressure; TG, triglyceride; LDL-C, lowdensity lipoprotein cholesterol; HDL-C, high-density lipoprotein cholesterol; Lp(a), lipoprotein a; Cr, creatinine; eGFR, estimated glomerular filtration rate; HbAlc, hemoglobin A1c; hsCRP, highsensitive c-reactive protein; baPWV, brachial-ankle pulse wave velocity; max-IMT, maximum intimamedia thickness; FMD, flow-mediated dilatation; ICTP, Carboxy-terminal telopeptide of type I collagen mean age 62.8) in this study. Fifty-three of 65 women were postmenopausal state. The clinical characteristics of the patient population are given in Table 1. Thirtysix patients were smoking (30.7\%); 71 had hypertension (60.6\%); 18 had diabetes mellitus (15.3\%); and 71 had dyslipidemia (60.6\%). All participants had at least one of the atherosclerotic risk factors described above; 16 patients had 3 risk factors; 47 had 2 risk factors; and 54 had 1 risk factor.

\section{Correlation of serum ICTP levels with max IMT val- ues, baPWV and FMD}

Serum ICTP levels were measured in all 117 patients, and found to be distributed between 1.6 and $7.5 \mathrm{ng} / \mathrm{mL}$ (mean $3.21 \pm 0.93 \mathrm{ng} / \mathrm{mL}$ ). Max IMT was ranged from 0.64 to $4.80 \mathrm{~mm}$ (mean $1.60 \pm 0.83 \mathrm{~mm}$ ); baPWV was from 987 to $2,748 \mathrm{~cm} / \mathrm{sec}$ (mean 1,513.1 $\pm 296.8 \mathrm{~cm} / \mathrm{sec}$ ); and FMD was from 0.2 to $10.3 \%$ (mean $4.36 \pm 2.11 \%$ ) (Table 1). Eighty-nine patients showed max IMT $\geq 1.1 \mathrm{~mm}$. The serum levels of ICTP were significantly higher in 89 patients with max IMT $\geq 1.1 \mathrm{~mm}$ than the remaining 28 patients with max IMT $<1.1 \mathrm{~mm}(3.33 \pm 0.97$ vs $2.82 \pm 0.65, p<0.05$, Fig. 1$)$. There were significant positive correlations between serum ICTP levels and max IMT ( $p<0.001$, Fig. 2A) or baPWV values ( $p<0.05$, Fig. $2 \mathrm{~B})$, but not with FMD $(p=0.367)$ by simple regression analysis.

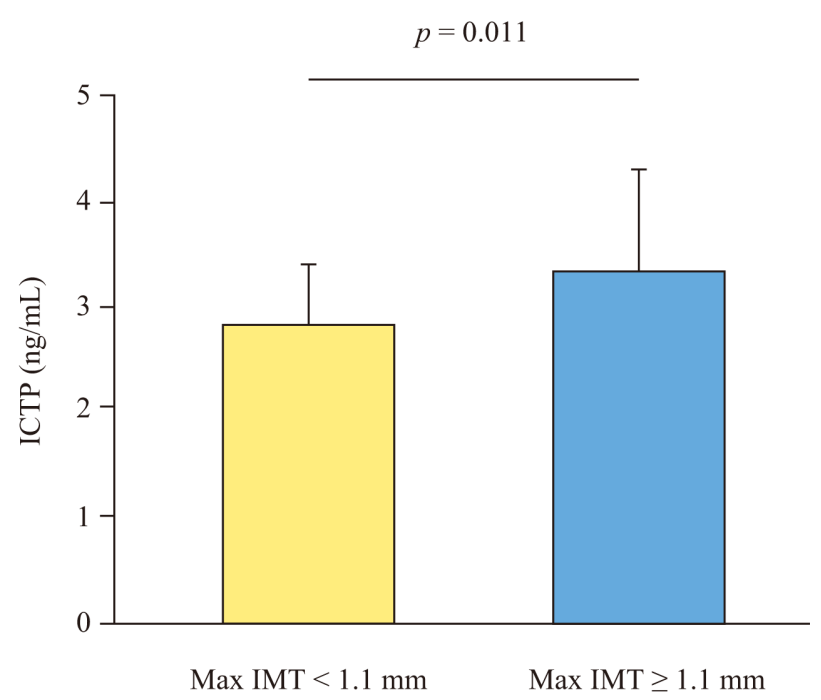

Fig. 1 Relationship between serum ICTP and max IMT Serum ICTP level was significantly higher in patients with max IMTs $\geq 1.1 \mathrm{~mm}$ compared to the patients with $\max$ IMTs $<1.1 \mathrm{~mm}(p<0.05)$. 

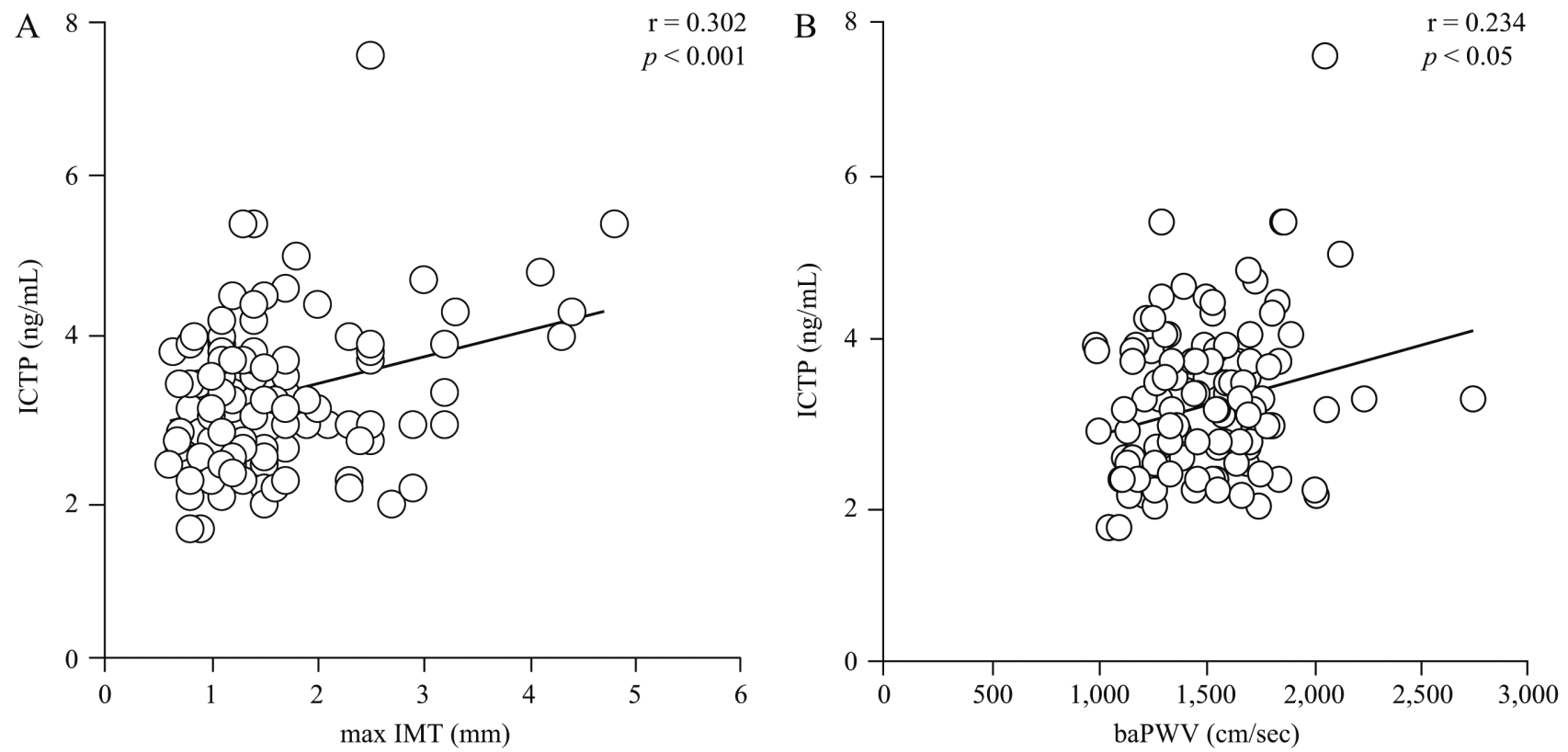

Fig. 2 Correlation between serum ICTP and max IMT (A), serum ICTP and baPWV (B)

Positive correlation was observed between serum ICTP and max IMT at $p<0.001$ and between serum ICTP and baPWV at $p<0.05$.

Factors correlated with max IMT, baPWV and FMD

Max IMT was positively correlated with age $(p<0.001)$, a male gender $(p<0.05)$, hypertension $(p<0.01)$, systolic blood pressure (SBP) $(p<0.05)$, $\mathrm{Lp}(\mathrm{a})(p<0.05)$, Cr $(p<0.01)$, hs-CRP $(p<0.05)$, and negatively correlated with HDL-C $(p<0.05)$. The value of baPWV was positively correlated with age $(p<0.001)$, hypertension $(p<0.001)$, SBP $(p<0.001)$, diastolic blood pressure (DBP) $(p<0.05)$, pulse pressure (PP) $(p<0.05)$ and hs-CRP $(p<0.01)$. FMD was positively correlated with HDL-C $(p<0.001)$, and negatively with hypertension $(p<0.01), \mathrm{HbA} 1 \mathrm{c}(p<0.05)$ by simple regression analysis (Table 2). Furthermore, multivariate analysis revealed that ICTP $(p<0.001)$, age $(p<0.05)$, and $\mathrm{Lp}(\mathrm{a})(p<0.05)$ were independently and positively associated with max IMT (Table 3 ). Again, ICTP $(p<0.05)$ and age $(p<0.001)$ were still significant determinants of max IMT when postmenopausal subjects were excluded (Supplementary table 1). On the other hand, multivariate analysis revealed that age $(p<0.001)$, SBP $(p<0.001)$, and hsCRP $(p<0.01)$ were independently associated with baPWV (Table 4). Although the significance of the correlation between baPWV and serum ICTP levels disappeared by multiple regression analysis, we found that the association between ICTP and baPWV was independent of SBP
(Table 5). Serum ICTP levels showed no significant relationship with the levels of bone metabolic markers, BSAP $(p=0.134)$ and NTX $(p=0.441)$. The values of max IMT and baPWV showed no significant relationship with $\operatorname{BSAP}(p=0.763, p=0.694$, respectively) or NTX ( $p=0.534, p=0.724$, respectively), either. Collectively, these results demonstrate that serum ICTP levels can serve as a surrogate marker for systemic atherosclerosis.

\section{Discussion}

In the present study, serum ICTP levels were demonstrated to be significantly higher in patients with max IMT $\geq 1.1 \mathrm{~mm}$ compared to those with max IMT $<1.1$ $\mathrm{mm}$. Significantly positive relationship between serum ICTP levels and max IMT or baPWV were observed by simple regression analysis. Furthermore, multivariate analysis revealed that serum ICTP levels were associated with an increment of max IMT. Although, there were no significant relationship between serum ICTP levels and baPWV values in multivariate analysis $(p=0.10)$, ICTP was still significant determinant of baPWV after adjusting SBP. As an atherosclerotic functional index, the decline of FMD could be reflected even in early stage of atherosclerosis [18]. In the pres- 
Table 2 Simple regression analysis for determinants of max IMT, baPWV and FMD

\begin{tabular}{|c|c|c|c|c|c|c|}
\hline & \multicolumn{2}{|c|}{ Max IMT } & \multicolumn{2}{|c|}{ baPWV } & \multicolumn{2}{|c|}{ FMD } \\
\hline & Coefficient & $p$ value & Coefficient & $p$ value & Coefficient & $p$ value \\
\hline Age & 0.400 & $<0.001$ & 0.447 & $<0.001$ & - & - \\
\hline Male gender & 0.226 & $<0.05$ & - & - & - & - \\
\hline BMI & - & - & - & - & - & - \\
\hline Current smoking & - & - & - & - & - & - \\
\hline Hypertension & 0.260 & $<0.01$ & 0.267 & $<0.01$ & -0.263 & $<0.01$ \\
\hline $\mathrm{DM}$ & - & - & - & - & - & - \\
\hline Dyslipidemia & - & - & - & - & - & - \\
\hline SBP & 0.193 & $<0.05$ & 0.422 & $<0.01$ & - & - \\
\hline DBP & - & - & 0.224 & $<0.05$ & - & - \\
\hline PP & - & - & 0.298 & $<0.01$ & - & - \\
\hline $\mathrm{HbAlc}$ & - & - & - & - & -0.194 & $<0.05$ \\
\hline TG & - & - & - & - & - & - \\
\hline LDL-C & - & - & - & - & - & - \\
\hline HDL-C & -0.205 & $<0.05$ & - & - & 0.323 & $<0.001$ \\
\hline $\mathrm{Lp}(\mathrm{a})$ & 0.224 & $<0.05$ & - & - & - & - \\
\hline $\mathrm{Cr}$ & 0.255 & $<0.01$ & - & - & - & - \\
\hline eGFR & - & - & - & - & - & - \\
\hline hsCRP & 0.215 & $<0.05$ & 0.249 & $<0.01$ & - & - \\
\hline ICTP & 0.302 & $<0.001$ & 0.234 & $<0.05$ & - & - \\
\hline
\end{tabular}

The abbreviations are the same as those in Table 1.

Table 3 Multiple regression analysis for determinants of max IMT

\begin{tabular}{lcccc}
\hline & $\beta$ & $95 \%$ CI & T value & $p$ value \\
\hline Age & 0.201 & 0.001 to 0.030 & 2.001 & $<0.05$ \\
Lp(a) & 0.385 & 0.001 to 0.019 & 2.329 & $<0.05$ \\
ICTP & 0.437 & 0.200 to 0.454 & 5.118 & $<0.001$ \\
\hline
\end{tabular}

Variables of the original model included the following: Age, male gender, BMI, current smoking, SBP, TG, LDL-C, HDL-C, Lp(a), $\mathrm{Cr}, \mathrm{HbAlc}$, hsCRP and ICTP. ICTP $(p<0.001)$, age $(p<0.05)$, and $\mathrm{Lp}(\mathrm{a})(p<0.05)$ were independently and positively associated with max IMT. The abbreviations are the same as those in Table 1.

ent study, FMD was negatively correlated with baPWV $(\mathrm{r}=-0.387, p<0.001)$ and max IMT $(\mathrm{r}=-0.238, p<0.01)$ in simple regression analysis. However, there was no correlation between FMD values and serum ICTP levels $(p=0.367)$. These findings suggested that serum ICTP levels were associated with vascular wall remodeling rather than endothelial layer remodeling in the process of the formation of atherosclerosis.

Atherosclerotic lesions, particularly with plaques, are rich in type I collagen, and macrophages are infiltrated into these lesions and abundantly produce MMPs $[10,19,20]$. Serum levels of MMPs are associated with a systemic atherosclerotic burden [21], and
Table 4 Multiple regression analysis for determinants of baPWV

\begin{tabular}{lcccc}
\hline & $\beta$ & $95 \%$ CI & T value & $p$ value \\
\hline Age & 0.590 & 12.002 to 19.361 & 8.434 & $<0.001$ \\
SBP & 0.392 & 4.244 to 8.177 & 5.858 & $<0.001$ \\
hsCRP & 0.222 & 16.598 to 94.564 & 2.828 & $<0.01$ \\
\hline
\end{tabular}

Variables of the original model included the following: Age, male gender, BMI, current smoking, SBP, LDL-C, HDL-C, Lp(a), Cr, HbAlc, hsCRP and ICTP. Age $(p<0.001)$, SBP $(p<0.001)$, and hsCRP $(p<0.01)$ were positively and independently associated with baPWV. The abbreviations are the same as those in Table 1.

Table 5 Multiple regression analysis of determinants for predictors of baPWV

\begin{tabular}{|c|c|c|c|c|}
\hline & \multicolumn{2}{|c|}{ Model 1} & \multicolumn{2}{|c|}{ Model 2} \\
\hline & $\beta$ & $p$ value & $\beta$ & $p$ value \\
\hline ICTP & 0.173 & 0.067 & 0.198 & $<0.05$ \\
\hline SBP & 0.204 & $<0.05$ & 0.427 & $<0.001$ \\
\hline Age & 0.56 & $<0.001$ & & \\
\hline
\end{tabular}

Model 1: ICTP, SBP and age were forced added as parameter for determinants of baPWV. Model 2: ICTP and SBP were forced added as parameter for determinants of baPWV. ICTP was still significant determinant of baPWV. The abbreviations are the same as those in Table 1. 
the expression of MMPs in progressive atherosclerotic lesions is markedly elevated [22]. ICTP is a MMPdependent degradation product of type I collagen, while other type I collagen degradation products such as NTX currently used as osteoclastic bone resorption markers are formed by cathepsin K-dependent degradation [19]. Metastatic bone lesions of cancers have been also known to cause an increase in serum ICTP levels through the cancer cell-derived MMP-dependent type I collagen degradation in the bone [23]. Because there was no significant correlation between the levels of serum ICTP and NTX in the present study, it is likely that ICTP is mainly derived from systemic atherosclerotic plaques rather than the age-related osteoporotic bone in our cohort. Our previous study also demonstrated that serum levels of ICTP was elevated in patients with a history of coronary symptoms and at least one coronary plaque with more than $50 \%$ stenosis [9]. However, mean ICTP concentration in our previous study was significantly higher than that of the present study $(7.22 \pm 10.10$ vs $3.21 \pm 0.93, p<0.001)$. The patients with coronary plaques in our previous study appear to have suffered from more advanced and systemic atherosclerosis than the patients of the present study. In fact, all patients enrolled in the present study did not experience any chest symptoms with normal ECG findings. This significant difference of serum ICTP levels between our previous and the present studies may depend on total amounts of atherosclerotic plaques or severity of systemic atherosclerosis.

Olsen et al. [24] showed that net synthesis of type I collagen was positively correlated with vascular hypertrophy and a blood pressure load in patients with essential hypertension. Their data suggested that collagen synthesis in vascular walls was increased in relation to a high hemodynamic load. Ishikawa et al. [25] showed that baPWV was positively correlated with serum lev- els of procollagen type I C-terminal propeptide (PICP) as well as ICTP and also with the ratios of serum levels of tissue inhibitor of matrix metalloproteinase-1 (TIMP-1) to MMP-1, indices of collagen turnover in hypertensive patients. This study indicated that collagen synthesis and degradation were increased in hypertensive subjects. MucNulty et al. [26] also reported that a type-I collagen synthesis to degradation ratio was negatively correlated with baPWV as well as an augmentation index, a marker of arterial stiffness, whereas serum MMP-1 levels displayed a positive correlation with both baPWV and an augmentation index. These studies collectively demonstrated the relationship between collagen metabolism and vascular stiffness or IMT.

Our study has limitations. First, this is a cross-sectional study with a relatively small sample size. Second, we were not able to assess if the patients were taking medications that would affect IMT and baPWV including statins, anti-hypertensives, and so forth. Third, we could not identify subjects with impaired glucose tolerance that is known to increase cardiovascular risks [27, 28]. Further studies on patients with a wider variety of conditions will be necessary to define the role of serum ICTP levels as a surrogate marker for the development of acute coronary syndrome, cerebrovascular accidents and peripheral arterial diseases.

Nonetheless, from the present study, serum ICTP levels appeared to be positively correlated with max IMT and baPWV values in patients who had at least one of atherosclerotic risk factors, and can serve as a useful non-invasive biomarker for assessment of systemic atherosclerosis.

\section{Disclosure}

The authors declare no conflicts of interest.

Supplementary Table 1 Multiple regression analysis of determinants for max IMT excluding postmenopausal women

\begin{tabular}{lcccc}
\hline & $\beta$ & $95 \%$ CI & T value & $p$ value \\
\hline Age & 0.440 & 0.015 to 0.048 & 3.783 & $<0.001$ \\
ICTP & 0.255 & 0.014 to 0.318 & 2.190 & $<0.05$ \\
\hline
\end{tabular}

Variables of the original model included the following: Age, male gender, BMI, current smoking, SBP, DBP, TG, LDL-C, HDL-C, Lp(a), Cr, HbAlc, hsCRP and ICTP. ICTP $(p<0.05)$ and age $(p<0.001)$ were still independently and positively associated with max IMT excluding postmenopausal women. The abbreviations are the same as those in Table 1. 


\section{References}

1. Cheng KS, Mikhailidis DP, Hamilton G, Seifalian AM (2002) A review of the carotid and femoral intimamedia thickness as an indicator of the presence of peripheral vascular disease and cardiovascular risk factors. Cardiovasc Res 54: 528-538.

2. O'Leary DH, Polak JF, Kronmal RA, Manolio TA, Burke GL, et al. (1999) Carotid-artery intima and media thickness as a risk factor for myocardial infarction and stroke in older adults. Cardiovascular Health Study Collaborative Research Group. $N$ Engl J Med 340: 14-22.

3. Baldassarre D, Amato M, Pustina L, Castelnuovo S, Sanvito S, et al. (2007) Measurement of carotid artery intima-media thickness in dyslipidemic patients increases the power of traditional risk factors to predict cardiovascular events. Atherosclerosis 191: 403-408.

4. Mattace-Raso FU, van der Cammen TJ, Hofman A, van Popele NM, Bos ML, et al. (2006) Arterial stiffness and risk of coronary heart disease and stroke: the Rotterdam Study. Circulation 113: 657-663.

5. O'Rourke MF, Staessen JA, Vlachopoulos C, Duprez D, Plante GE (2002) Clinical applications of arterial stiffness; definitions and reference values. Am J Hypertens 15: 426-444.

6. Shekhonin BV, Domogatsky SP, Muzykantov VR, Idelson GL, Rukosuev VS (1985) Distribution of type I, III, IV and V collagen in normal and atherosclerotic human arterial wall: immunomorphological characteristics. Coll Relat Res 5: 355-368.

7. Fornieri C, Quaglino D Jr, Mori G (1992) Role of the extracellular matrix in age-related modifications of the rat aorta. Ultrastructural, morphometric, and enzymatic evaluations. Arterioscler Thromb 12: 1008-1016.

8. Sundström J, Vasan RS (2006) Circulating biomarkers of extracellular matrix remodeling and risk of atherosclerotic events. Curr Opin Lipidol 17: 45-53.

9. Kato S, Endo I, Fujimura M, Kuriwaka-Kido R, Fujinaka Y, et al. (2013) Serum carboxy-terminal telopeptide of type I collagen (ICTP) as a surrogate marker for vulnerable plaques in atherosclerotic patients: a pilot study. Athrosclerosis 229: 182-185.

10. Loftus IM, Naylor AR, Goodall S, Crowther M, Jones L, et al. (2000) Increased matrix metalloproteinase-9 activity in unstable carotid plaques. A potential role in acute plaque disruption. Stroke 31: 40-47.

11. Ducharme A, Frantz S, Aikawa M, Rabkin E, Lindsey M, et al. (2000) Targeted deletion of matrix metalloproteinase-9 attenuates left ventricular enlargement and collagen accumulation after experimental myocardial infarction. J Clin Invest 106: 55-62.

12. Ter Avest E, Stalenhoef AF, de Graaf J (2007) What is the role of non-invasive measurements of atherosclero$\mathrm{sis}$ in individual cardiovascular risk prediction? Clin $\mathrm{Sci}$
(Lond) 112: 507-516.

13. Simon A, Chironi G, Levenson J (2007) Comparative performance of subclinical atherosclerosis tests in predicting coronary heart disease in asymptomatic individuals. Eur Heart J 28: 2967-2971.

14. Tomiyama H, Hashimoto H, Tanaka H, Matsumoto C, Odaira M, et al. (2010) Continuous smoking and progression of arterial stiffening: A prospective study. $\mathrm{J} \mathrm{Am}$ Coll Cardiol 55: 1979-1987.

15. Tomiyama H, Hashimoto H, Tanaka H, Matsumoto C, Odaira M, et al. (2010) Synergistic relationship between changes in the pulse wave velocity and changes in the heart rate in middle-aged japanese adults: A prospective study. J Hypertens 28: 687-694.

16. Yoshida S, Aihara K, Azuma H, Uemoto R, SumitomoUeda Y, et al. (2010) Dehydroepiandrosterone sulfate is inversely associated with sex-dependent diverse carotid atherosclerosis regardless of endothelial function. Atherosclerosis 212: 310-315.

17. Aihara K, Azuma H, Takamori N, Kanagawa Y, Akaike M, et al. (2004) Heparin cofactor II is a novel protective factor against carotid atherosclerosis in elderly individuals. Circulation 109: 2761-2765.

18. Ciccone MM, Bilianou E, Balbarini A, Gesualdo M, Ghiadoni L, et al. (2013) Task force on: 'Early markers of atherosclerosis: influence of age and sex'. $J$ Cardiovasc Med (Hagerstown) 14: 757-766.

19. Garnero P, Ferreras M, Karsdal MA, Nicamhlaoibh R, Risteli J, et al. (2003) The type I collagen fragments ICTP and CTX reveal distinct enzymatic pathways of bone collagen degradation. J Bone Miner Res 18: 859-867.

20. Visse R, Nagase H (2003) Matrix metalloproteinases and tissue inhibitors of metalloproteinases: structure, function, and biochemistry. Circ Res 92: 827-839.

21. Goncalves I, Bengtsson E, Colhoun HM, Shore AC, Palombo C, et al. (2015) Elevated Plasma Levels of MMP-12 Are Associated With Atherosclerotic Burden and Symptomatic Cardiovascular Disease in Subjects With Type 2 Diabetes. Arterioscler Thromb Vasc Biol 35: 1723-1731.

22. Cheng C, Tempel D, van Haperen R, van Damme L, Algür M, et al. (2009) Activation of MMP8 and MMP13 by angiotensin II correlates to severe intra-plaque hemorrhages and collagen breakdown in atherosclerotic lesions with a vulnerable phenotype. Atherosclerosis 204: 26-33.

23. Koizumi M, Takahashi S, Ogata E (2003) Comparison of serum bone resorption markers in the diagnosis of skeletal metastasis. Anticancer Res 23: 4095-4099.

24. Olsen MH, Christensen MK, Wachtell K, Tuxen C, Fossum E, et al. (2005) Markers of collagen synthesis is related to blood pressure and vascular hypertrophy: a 
LIFE substudy. J Hum Hypertens 19: 301-307.

25. Ishikawa J, Kario K, Matsui Y, Shibasaki S, Morinari $\mathrm{M}$, et al. (2005) Collagen metabolism in extracellular matrix may be involved in arterial stiffness in older hypertensive patients with left ventricular hypertrophy. Hypertens Res 28: 995-1001.

26. McNulty M, Mahmud A, Spiers P, Feely J (2006) Collagen type-I degradation is related to arterial stiffness in hypertensive and normotensive subjects. J Hum
Hypertens 20: 867-873.

27. Lee M, Saver JL, Hong KS, Song S, Chang KH, et al. (2012) Effect of pre-diabetes on future risk of stroke: Meta-analysis. BMJ 344: e3564.

28. DeFronzo RA, Abdul-Ghani M (2011) Assessment and treatment of cardiovascular risk in prediabetes: Impaired glucose tolerance and impaired fasting glucose. Am J Cardiol 108 (3 Suppl): 3B-24B. 